\title{
Construction of Optimal Sets of Frequency Hopping Sequences
}

\author{
Bin Wen \\ Department of Mathematics, Changshu Institute of Technology, Changshu, Jiangsu 215500, China \\ Correspondence should be addressed to Bin Wen; wenbin9903@163.com
}

Received 18 December 2012; Accepted 3 January 2013

Academic Editors: C. da Fonseca and A. V. Kelarev

Copyright (C) 2013 Bin Wen. This is an open access article distributed under the Creative Commons Attribution License, which permits unrestricted use, distribution, and reproduction in any medium, provided the original work is properly cited.

Frequency hopping spread spectrum and direct sequence spread spectrum are two main spread coding technologies. Frequency hopping sequences are needed in FH-CDMA systems. In this paper, a construction of optimal sets of frequency hopping sequences is presented. The construction is based on the set-theoretic characterization of an optimal set of $\mathrm{FH}$ sequences.

\section{Introduction}

Frequency hopping spread spectrum and direct sequence spread spectrum are two main spread coding technologies. Frequency hopping sequences are an integral part of spreadspectrum communication systems such as FH-CDMA systems (for a description of such systems, see [1]). In modern radar and communication systems, frequency-hopping $(\mathrm{FH})$ spread-spectrum techniques have become popular (see [2], for example).

Assume that $F=\left\{f_{0}, f_{1}, \ldots, f_{m-1}\right\}$ is a set of available frequencies, called an alphabet. Let $\mathscr{X}(v ; F)$ be the set of all sequences of length $v$ over $F$. Any element of $\mathscr{X}(v ; F)$ is called a frequency hopping sequence (FHS) of length $v$ over $F$. Given two FH sequences, $X=\left(x_{0}, x_{1}, \ldots, x_{v-1}\right)$ and $Y=\left(y_{0}, y_{1}, \ldots, y_{v-1}\right)$, we define their Hamming correlation $H_{X, Y}(w)$ to be

$$
H_{X, Y}(w)=\sum_{0 \leq i \leq v-1} h\left[x_{i}, y_{i+w}\right]
$$

where $0 \leq w<v$ if $X \neq Y$ and $0<w<v$ if $X=Y$, and where

$$
h[x, y]= \begin{cases}1, & \text { if } x=y \\ 0, & \text { otherwise }\end{cases}
$$

and all operations among position indices are performed modulo $v$. If $X=Y, H_{X, Y}(w)$ is the Hamming autocorrelation. If $X \neq Y, H_{X, Y}(w)$ is the Hamming cross correlation.

\section{Lower Bounds on the Correlations of FHSs}

FH sequences for FH-CDMA systems are required to have good Hamming correlations and large linear span [3]; the linear span is defined to be the length of the shortest linear feedback shift register that can produce the sequence. $\mathrm{FH}$ sequences' design normally involves six parameters: the size $m$ of the frequency library $F$, the sequence length $v$, the family size $N$ of the subset $\mathcal{S} \subset \mathscr{X}(v ; F)$, the maximum out-of-phase Hamming autocorrelations $H_{a}$, the maximum Hamming cross correlations $H_{c}$, and the linear span. It is generally desired that the family $\delta$ of $\mathrm{FH}$ sequences has the following properties:

(1) the maximum out-of-phase Hamming autocorrelations $H_{a}$ should be as small as possible,

(2) the maximum Hamming cross correlations $H_{c}$ should be as small as possible,

(3) the family size $N=|\delta|$ for given $H_{a}, H_{c}, M$, and $v$ should be as large as possible,

(4) the linear span should be as large as possible.

In order to evaluate the theoretical performance of the $\mathrm{FH}$ sequences, it is important to find some theoretical bounds for these parameters. Given $m, v$, and $N$ of $\mathcal{S}$, Lempel and Greenberger [4] and Peng and Fan [5] derived lower bounds on $H_{a}$ and $H_{c}$ of $\mathrm{FH}$ sequences in $\mathscr{X}(v ; F)$. We restate their results in this section, which will be used later as the criteria to determine whether the new FH sequences constructed in this paper are optimal or not. 
For any single FH sequence $X \in \mathscr{X}(v ; F)$, let $H_{a}(X)=$ $\max _{1 \leq t \leq v-1}\left\{H_{X, X}(t)\right\}$ be the maximum out-of-phase value of $H_{X, X}(t)$. If $H_{a}\left(X^{*}\right) \leq H_{a}(X)$ for all $X \in \mathscr{X}(v ; F)$, that is, if the value $H_{a}\left(X^{*}\right)$ is the least among all $\mathrm{FH}$ sequences of the same length $v$ and over the same frequency library $F, X^{*}$ is called an optimal FH sequence. Lempel and Greenberger [4] developed the following lower bound for $H_{a}(X)$.

Lemma 1 (see [4]). For every FH sequence $X$ of length $v$ over a frequency alphabet $F$ of size $m$. One has

$$
H_{a}(X) \geq \frac{(v-\epsilon)(v+\epsilon-m)}{m(v-1)},
$$

where $\epsilon$ is the least nonnegative residue of $v$ modulo $m$.

Corollary 2. For every FH sequence $X$ of length $v$ over a frequency alphabet $F$ of size $m$. One has

$$
H_{a}(X) \geq \begin{cases}k, & \text { if } m \neq v, \\ 0, & \text { if } m=v,\end{cases}
$$

where $v=k m+\varepsilon$ with $0 \leq \varepsilon \leq m-1$.

For any given subset $\mathcal{S}$ of $\mathscr{X}(v ; F)$ containing $N$ FH sequences, any two $X, Y \in \mathcal{S}, X \neq Y$, we write $H_{c}(X, Y)=$ $\max _{0 \leq t \leq v-1}\left\{H_{X, Y}(t)\right\}$. We define the maximum out-of-phase Hamming autocorrelations $H_{a}(\mathcal{S})$ and the maximum Hamming cross correlations $H_{c}(\mathcal{S})$ as

$$
\begin{aligned}
& H_{a}(\mathcal{S})=\max \left\{H_{a}(X): X \in \mathcal{S}\right\} \\
& H_{c}(\mathcal{S})=\max \left\{H_{c}(X, Y): X, Y \in \mathcal{S}, X \neq Y\right\} \\
& M(\mathcal{S})=\max \left\{H_{a}(\mathcal{S}), H_{c}(\mathcal{S})\right\} .
\end{aligned}
$$

Peng and Fan [5] developed the following bounds on $M(\mathcal{S})$, which take into consideration the number of $\mathrm{FH}$ sequences in the set.

Lemma 3 (see [5]). Let $\mathcal{S} \subseteq \mathscr{X}(v ; F)$ be a set of $N$ sequences of length $v$ over an alphabet of size $m$. Define $I=\lfloor v N / m\rfloor$. Then

$$
\begin{aligned}
& M(\mathcal{S}) \geq\left\lceil\frac{(v N-m) v}{(v N-1) m}\right\rceil, \\
& M(\mathcal{S}) \geq\left\lceil\frac{2 I v N-(I+1) m I}{(v N-1) N}\right\rceil .
\end{aligned}
$$

A family $\mathcal{S} \subseteq \mathscr{X}(v ; F)$ is an optimal set if the Peng-Fan lower bound (2) or (3) in Lemma 3 is met. Let $N=m$, and we have the following corollary which is a useful tool to check the bound in Lemma 3.

Corollary 4 (see $[6])$. Let $\mathcal{S} \subseteq \mathscr{X}(v ; F)$ be a set of $m$ sequences of length $v$ over an alphabet of size $m$. Then

$$
M(\mathcal{S}) \geq \begin{cases}a, & \text { if } m \mid v, \\ a+1, & \text { if } m+v,\end{cases}
$$

where $v=a m+b$ with $0 \leq b \leq m-1$.
A number of authors have made contributions to the construction of optimal FH sequences. Both algebraic and combinatorial constructions of optimal $\mathrm{FH}$ sequences have been given (see, for example, $[3,4,7-11]$ ). Most of them are concentrated on single optimal FH sequences. The purpose of this paper is to present a construction of optimal sets of $\mathrm{FH}$ sequences based on the set-theoretic characterization of an optimal set of FH sequences. Throughout what follows, we use $(v, m, \lambda)$-FHS to denote an FH sequence $X$ of length $v$ over an alphabet of size $m$ whose Hamming autocorrelation $H_{a}(X)=\lambda$. We also call a set $\mathcal{S}$ of $N \mathrm{FH}$ sequences in $\mathscr{X}(v ; F)$ a $(v, N, \lambda ; m)$ set of $\mathrm{FH}$ sequences, where $\lambda=M(\mathcal{S})$.

\section{Mixed Difference Functions}

Fuji-Hara et al. [9] characterized a $(v, m, \lambda)$-FHS in terms of partition-type cyclic difference packings. Given a partition $\mathscr{D}=\left\{D_{0}, D_{1}, \ldots, D_{m-1}\right\}$ of $Z_{v}$ into $m$ subsets (called base blocks), we can define a difference function on $\mathbb{Z}_{v}^{*}=\mathbb{Z}_{v} \backslash\{0\}$ given by $\Gamma_{\mathscr{D}}(w)=\sum_{i=0}^{m-1}\left|\left(D_{i}+w\right) \cap D_{i}\right|$. Let $\max \left\{\Gamma_{\mathscr{D}}(w) \mid w \in\right.$ $\left.\mathbb{Z}_{v} \backslash\{0\}\right\}=\lambda$. Then $\mathscr{D}$ is called a $(v, K, \lambda)_{m}$-PCDP (partitiontype cyclic difference packing). Here, $m$ is used in the notation to indicate the number of base blocks, and $K=\left\{\left|D_{i}\right|: 0 \leq\right.$ $i \leq m-1\}$ is the list of the sizes of base blocks. This is to say that a $(v, K, \lambda)_{m}$-PCDP is a partition of $\mathbb{Z}_{v}$ into $m$ base blocks which satisfies the following property. For any fixed nonzero residue $w \in \mathbb{Z}_{v}$, the equation $x-y=w$ has at most $\lambda$ solutions $(x, y)$ in the multiset union $\bigcup_{D \in \mathscr{D}}(D \times D)$.

If we label the positions of a $(v, m, \lambda)$-FHS $X$ by the elements of $\mathbb{Z}_{v}$, then, by the above definition, the sets of position indices of $m$ frequencies in $X$ form a $(v, K, \lambda)_{m^{-}}$ PCDP $\mathscr{D}$ with $\Gamma_{\mathscr{D}}(w)=H_{X, X}(w)$ for any nonzero $w \in \mathbb{Z}_{v}$. Conversely, if we label the $m$ base blocks of a $(v, K, \lambda)_{m^{-}}$ PCDP by the elements of $\mathbb{Z}_{m}$ and identify the frequency alphabet $F$ with $\mathbb{Z}_{m}$, then the PCDP gives a $(v, m, \lambda)$-FHS in $\mathscr{X}(v ; F)$.

This fact reveals that a single FHS can be constructed by a PCDP. Apparently, the smaller the index $\lambda$ of a PCDP the lower the Hamming autocorrelation $H_{a}(X)$ of its corresponding $\mathrm{FH}$ sequence. For an optimal $\mathrm{FH}$ sequence, we need to construct a $(v, K, \lambda)_{m}$-PCDP so that its index $\lambda$ is as small as possible for any given value of $v$ and $m$. Based on Lempel-Greenberger bound on $H_{a}(X)$ in Lemma 1, FujiHara et al. [9] proved the following result.

Lemma 5 (see [9]). There exists a $(v, m, \lambda)$-FHS over the alphabet $F=\mathbb{Z}_{m}$ if and only if there exists $a(v, K, \lambda)_{m}-P C D P$ in $\mathbb{Z}_{v}$. Furthermore, this FH sequence is optimal if $\lambda=\lfloor v / m\rfloor$ for $v>m$ and if $\lambda=0$ for $v=m$.

The correspondence between an individual FH sequence and a PCDP can be naturally extended to give a set-theoretic interpretation of a set of $\mathrm{FH}$ sequences. Let $N$ be a positive integer. Let $\mathscr{C}=\left\{\mathscr{D}^{(0)}, \mathscr{D}^{(1)}, \ldots, \mathscr{D}^{(N-1)}\right\}$ be a collection of partitions of $\mathbb{Z}_{v}$ into $m$ subsets (called base blocks). Write $\mathscr{D}^{(r)}=\left\{D_{0}^{(r)}, D_{1}^{(r)}, \ldots, D_{m-1}^{(r)}\right\}, 0 \leq r \leq N-1$. For any ordered pair $(i, j)$ with $0 \leq i<j \leq N-1$, we define 
a difference function on $\mathbb{Z}_{v}$ given by $\Gamma_{\mathscr{C}}^{(i, j)}(w)=\sum_{k=0}^{m-1} \mid\left(D_{k}^{(i)}+\right.$ $w) \bigcap D_{k}^{(j)} \mid$. For any integer $r$ with $0 \leq r \leq N-1$, we define a difference function on $\mathbb{Z}_{v} \backslash\{0\}$, as before, given by $\Gamma_{\mathscr{C}}^{(r, r)}(w)=\Gamma_{\mathscr{D}^{(r)}}(w)=\sum_{k=0}^{m-1}\left|\left(D_{k}^{(r)}+w\right) \cap D_{k}^{(r)}\right|$. We refer to these $N(N+1) / 2$ difference functions defined above as mixed difference functions with respect to the given collection $\mathscr{C}$. Since each partition in $\mathscr{C}$ determines uniquely an $\mathrm{FH}$ sequence, the collection $\mathscr{C}$ gives a set of $N$ FH sequences in $\mathscr{X}(v ; F)$, and vice versa, where the alphabet is regarded as $\mathbb{Z}_{m}$. For the optimality of the derived set of $\mathrm{FH}$ sequences from $\mathscr{C}$, we define $\lambda(r)=\max _{1 \leq w<v}\left\{\Gamma_{\mathscr{C}}^{(r, r)}(w)\right\}, 0 \leq r \leq$ $N-1, \lambda(i, j)=\max _{0 \leq w<v}\left\{\Gamma_{\mathscr{C}}^{(i, j)}(w)\right\}, 0 \leq i<j \leq N-1$, $\mu(i, j)=\max \{\lambda(i), \lambda(j), \lambda(i, j)\}, 0 \leq i<j \leq N-1$, $\lambda=\max \left\{\max _{0 \leq r \leq N-1} \lambda(r), \max _{0 \leq i<j \leq N-1} \lambda(i, j)\right\}$. Then $\mathscr{D}^{(r)}$ is a $\left(v, K_{r}, \lambda_{r}\right)_{m}$-PCDP, according to the previous definition. We say that $\mathscr{C}$ is a $\left(v,\left\{K_{0}, K_{1}, \ldots, K_{N-1}\right\},\left\{\lambda_{0}, \lambda_{1}, \ldots, \lambda_{N-1}\right\} ; \lambda\right)_{m}$ collection of $N$ PCDPs. It turns out that there exists a $(v, N, \lambda ; m)$ set of FH sequences in $\mathscr{X}(v ; F)$ if and only if there exists a $\left(v,\left\{K_{0}, K_{1}, \ldots, K_{N-1}\right\},\left\{\lambda_{0}, \lambda_{1}, \ldots, \lambda_{N-1}\right\} ; \lambda\right)_{m}$ collection of $N$ PCDPs in $Z_{v}$ under our notations. This gives us an interpretation for a set of $\mathrm{FH}$ sequences from set-theoretic perspective. As with individual optimal $\mathrm{FH}$ sequence, for an optimal $(v, N, \lambda ; m)$ set of $\mathrm{FH}$ sequences, we are required to construct a $\left(v,\left\{K_{0}, K_{1}, \ldots, K_{N-1}\right\},\left\{\lambda_{0}, \lambda_{1}, \ldots, \lambda_{N-1}\right\} ; \lambda\right)_{m}$ collection $\mathscr{C}$ of $N$ PCDPs so that its index $\lambda$ is as small as possible. Since the index $\lambda$ of $\mathscr{C}$ is the same as the Hamming correlation $M(\mathcal{S})$, the Peng-Fan bounds in Lemma 3 can be employed as our benchmarks. As noted in [12], a set of FH sequences meeting one of the Peng-Fan bounds must be optimal. We have the following theorem.

Theorem 6 (see [13]). Let $N \geq 2$ be an integer. Then there exists $a(v, N, \lambda ; m)$ set of FH sequences in $\mathscr{X}(v ; F)$ if and only if there exists $a\left(v,\left\{K_{0}, K_{1}, \ldots, K_{N-1}\right\},\left\{\lambda_{0}, \lambda_{1}, \ldots, \lambda_{N-1}\right\} ; \lambda\right)_{m}$ collection of $N$ PCDPs in $\mathbb{Z}_{v}$. Furthermore, this set is optimal if $\lambda$ meets one of the Peng-Fan lower bounds given in Lemma 3.

\section{The Construction of Optimal Sets of FH Sequences}

To begin with, we state some preparatory knowledge that will be used in our constructions.

Let $q=p^{s}$ be a power of prime $p$, where $s$ is an arbitrary positive integer. Write $\operatorname{Tr}_{q / p}$ for the absolute trace function from $\mathbb{F}_{q}$ to $\mathbb{F}_{p}$ defined by

$$
\operatorname{Tr}_{q / p}(x)=x+x^{p}+x^{p^{2}}+\cdots+x^{p^{s-1}}
$$

We will use the following known result.

Lemma 7 (see [14, Corollary 7.17]). For any $a \in \mathbb{F}_{q}$ the number of elements $\alpha \in \mathbb{F}_{q^{m}}$ such that $\operatorname{Tr}_{q^{m} / q}(\alpha)=a$ is $q^{m-1}$.

With the previous preparations, we are now ready to describe our construction of optimal sets of FH sequences.
Theorem 8. Let $p$ be an odd prime and $m$ a positive integer. Define $f: \mathbb{Z}_{p^{m}-1} \rightarrow \mathbb{Z}_{p}$ by $f(x)=\operatorname{Tr}_{p^{m} / p}\left(\alpha^{x}\right)$, where $\operatorname{Tr}_{p^{m} / p}$ is the trace function from $\mathbb{F}_{p^{m}}$ to $\mathbb{F}_{p}$, $\alpha$ is a generator of $\mathbb{F}_{p^{m}}^{*}$. Define

$$
\begin{aligned}
D_{j} & =f^{-1}(j)=\left\{x \in \mathbb{Z}_{p^{m}-1}: f(x)=j\right\}, \quad 0 \leq j \leq p-1, \\
D_{j}^{(r)} & =D_{j+r}, \quad 0 \leq j \leq p-1,0 \leq r \leq p-1, \\
\mathscr{D}^{(r)} & =\left\{D_{0}^{(r)}, D_{1}^{(r)}, \ldots, D_{p-1}^{(r)}\right\}, \quad 0 \leq r \leq p-1,
\end{aligned}
$$

where all operations among the subscripts are performed modulo $p$. Then $\mathscr{C}=\left\{\mathscr{D}^{(0)}, \mathscr{D}^{(1)}, \ldots, \mathscr{D}^{(p-1)}\right\}$ is an optimal $\left(p^{m}-1, p, p^{m-1} ; p\right)$ set of FH sequences over $\mathbb{Z}_{p}$ oflength $p^{m}-1$. Furthermore, each sequence of the set $\mathscr{C}$ is optimal with respect to the bound of Lemma 1.

Proof. For any $r$ with $0 \leq r \leq e-1$ and any $w \in \mathbb{Z}_{p^{m}-1}^{*}$, we have

$$
\begin{aligned}
\Gamma_{\mathscr{C}}^{(r, r)}(w) & =\sum_{k=0}^{p-1}\left|\left(D_{k}^{(r)}+w\right) \bigcap D_{k}^{(r)}\right| \\
& =\left|\bigcup_{k=0}^{p-1}\left(\left(D_{k+r}+w\right) \bigcap D_{k+r}\right)\right| \\
& =\left|\bigcup_{k=0}^{p-1}\left(\left(D_{k}+w\right) \bigcap D_{k}\right)\right| \\
& =\left|\left\{x \in \mathbb{Z}_{p^{m}-1} \mid f(x+w)-f(x)=0\right\}\right| \\
& =\left|\left\{x \in \mathbb{Z}_{p^{m}-1} \mid \operatorname{Tr}_{p^{m} / p}\left[\left(\alpha^{w}-1\right) \alpha^{x}\right]=0\right\}\right| \\
& =p^{m-1}-1,
\end{aligned}
$$

where the last equality follows from Lemma 7 . Next we will show that $\Gamma_{\mathscr{C}}^{(i, j)}(w)=p^{m-1}$ for any $i, j$ with $0 \leq i<j \leq p-1$ and any $w \in \mathbb{Z}_{p^{m}-1}^{*}$. For any $i, j$ with $0 \leq i<j \leq e-1$ and any $w \in \mathbb{Z}_{p^{m}-1}^{*}$, we have

$$
\begin{aligned}
\Gamma_{\mathscr{C}}^{(i, j)}(w) & =\sum_{k=0}^{e-1}\left|\left(D_{k}^{(i)}+w\right) \bigcap D_{k}^{(j)}\right| \\
& =\sum_{k=0}^{e-1}\left|\left(D_{k+i}+w\right) \bigcap D_{k+j}\right| \\
& =\sum_{k=0}^{e-1}\left|\left(D_{k+i}+w\right) \bigcap D_{(k+i)+(j-i)}\right| \\
& =\sum_{k=0}^{e-1}\left|\left(D_{k}+w\right) \bigcap D_{k+(j-i)}\right| \\
& =\sum_{k=0}^{e-1}\left|\left(D_{k}+w\right) \bigcap D_{k+b}\right|, \quad b=j-i
\end{aligned}
$$




$$
\begin{aligned}
& =\left|\left\{x \in \mathbb{Z}_{p^{m}-1} \mid f(x+w)-f(x)=b\right\}\right| \\
& =\left|\left\{x \in \mathbb{Z}_{p^{m}-1} \mid \operatorname{Tr}_{p^{m} / p}\left[\left(\alpha^{w}-1\right) \alpha^{x}\right]=b\right\}\right| \\
& =p^{m-1},
\end{aligned}
$$

where the last equality follows from Lemma 7. It follows that $\mathscr{C}$ is a $\left(p^{m}-1,\left\{K_{0}, K_{1}, \ldots, K_{p-1}\right\},\left\{\lambda_{0}, \lambda_{1}, \ldots, \lambda_{p-1}\right\} ; p^{m-1}\right)_{p}$ collection of $p$ PCDPs in $\mathbb{Z}_{p}$, where $\lambda_{0}=\lambda_{1}=\cdots=\lambda_{m-1}=$ $p^{m-1}-1$. By Theorem 6 , it derives a $\left(p^{m}-1, p, p^{m-1} ; p\right)$ set of FH sequences in $\mathscr{X}(v ; F)$. For this set, we have $N=p$ and both lower bounds in Lemma 3 are equal to $p^{m-1}$ by Corollary 4. Hence, it is optimal. Furthermore, each sequence of the set $\mathscr{C}$ is optimal with respect to the bound of Lemma 1. The proof is then complete.

Example 9. Take $p=3$ and $m=2$. It is readily calculated that

$$
D_{0}=\{2,6\}, \quad D_{1}=\{1,3,4\}, \quad D_{2}=\{0,5,7\} .
$$

We then have

$$
\begin{aligned}
& \mathscr{D}^{(0)}=\left\{D_{0}^{(0)}=\{2,6\}, D_{1}^{(0)}=\{1,3,4\}, D_{2}^{(0)}=\{0,5,7\}\right\}, \\
& \mathscr{D}^{(1)}=\left\{D_{0}^{(1)}=\{1,3,4\}, D_{1}^{(1)}=\{0,5,7\}, D_{2}^{(1)}=\{2,6\}\right\}, \\
& \mathscr{D}^{(2)}=\left\{D_{0}^{(2)}=\{0,5,7\}, D_{1}^{(2)}=\{2,6\}, D_{2}^{(2)}=\{1,3,4\}\right\},
\end{aligned}
$$

The family $\mathscr{C}=\left\{\mathscr{D}^{(0)}, \mathscr{D}^{(1)}, \mathscr{D}^{(2)}\right\}$ is a $\left(v, m,\left\{K_{0}, K_{1}\right.\right.$, $\left.\left.K_{2}\right\} ;\left\{\lambda_{0}, \lambda_{1}, \lambda_{2}\right\}\right)_{3}$ collection of 3 PCDPs in $Z_{9}$, where $K_{0}=$ $\{2,3,3\}, K_{1}=\{3,3,2\}, K_{2}=\{3,2,3\}$, and $\lambda_{0}=\lambda_{1}=\lambda_{2}=2$. By Theorem 6 , it corresponds a $(8,3,3 ; 3)$ set $\delta$ consisting of the following $3 \mathrm{FH}$ sequences over $Z_{3}$ :

$$
\begin{aligned}
& X=(2,1,0,1,1,2,0,2), \\
& Y=(1,0,2,0,0,1,2,1), \\
& Z=(0,2,1,2,2,0,1,0),
\end{aligned}
$$

with $M(\mathscr{C})=3$. It is optimal meeting the lower bounds in Lemma 3.

\section{Acknowledgment}

The Paper was supported by the Tian Yuan Special Funds of the National Natural Science Foundation of China (Grant no. 11226282).

\section{References}

[1] R. A. Scholtz, "Spread spectrum concept," IEEE Transactions on Communications, vol. 25, no. 8, pp. 748-755, 1977.

[2] P. Fan and M. Darnell, Sequence Design for Communications Applications, Research Studies Press, Taunton, UK, 1996.

[3] P. V. Kumar, "Frequency-hopping code sequence designs having large linear span," IEEE Transactions on Information Theory, vol. 34, no. 1, pp. 146-151, 1988.
[4] A. Lempel and H. Greenberger, "Families of sequences with optimal Hamming correlation properties," IEEE Transactions on Information Theory, vol. IT-20, pp. 90-94, 1974.

[5] D. Peng and P. Fan, "Lower bounds on the Hamming auto- and cross correlations of frequency-hopping sequences," IEEE Transactions on Information Theory, vol. 50, no. 9, pp. 2149-2154, 2004.

[6] B. Wen, Y. Li, and C. Shi, "A construction of optimal sets of FH sequences," The Australasian Journal of Combinatorics, vol. 50, pp. 37-44, 2011.

[7] W. Chu and C. J. Colbourn, "Optimal frequency-hopping sequences via cyclotomy," IEEE Transactions on Information Theory, vol. 51, no. 3, pp. 1139-1141, 2005.

[8] C. Ding, M. J. Moisio, and J. Yuan, "Algebraic constructions of optimal frequency-hopping sequences," IEEE Transactions on Information Theory, vol. 53, no. 7, pp. 2606-2610, 2007.

[9] R. Fuji-Hara, Y. Miao, and M. Mishima, "Optimal frequency hopping sequences: a combinatorial approach," IEEE Transactions on Information Theory, vol. 50, no. 10, pp. 2408-2420, 2004.

[10] G. Ge, R. Fuji-Hara, and Y. Miao, "Further combinatorial constructions for optimal frequency-hopping sequences," Journal of Combinatorial Theory A, vol. 113, no. 8, pp. 1699-1718, 2006.

[11] P. Udaya and M. U. Siddiqi, "Optimal large linear complexity frequency hopping patterns derived from polynomial residue class rings," IEEE Transactions on Information Theory, vol. 44, no. 4, pp. 1492-1503, 1998.

[12] C. Ding and J. Yin, "Sets of optimal frequency-hopping sequences," IEEE Transactions on Information Theory, vol. 54, no. 8, pp. 3741-3745, 2008.

[13] J. Yin, "A construction of optimal sets of FH sequences," in Coding and Cryptology, vol. 4 of Series on Coding Theory and Cryptology, pp. 268-276, World Scientific, Hackensack, NJ, USA, 2008.

[14] Z.-X. Wan, Lectures on Finite Fields and Galois Rings, World Scientific Publishing, River Edge, NJ, USA, 2003. 


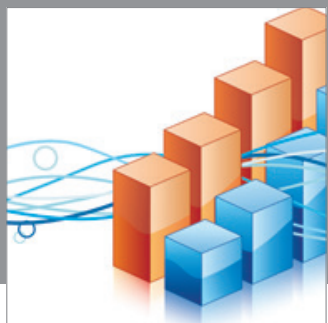

Advances in

Operations Research

mansans

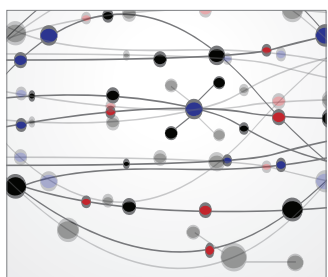

The Scientific World Journal
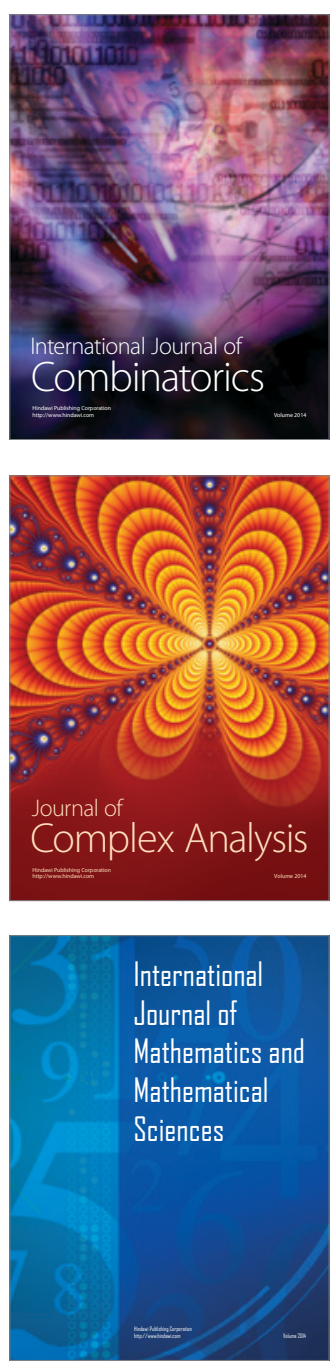
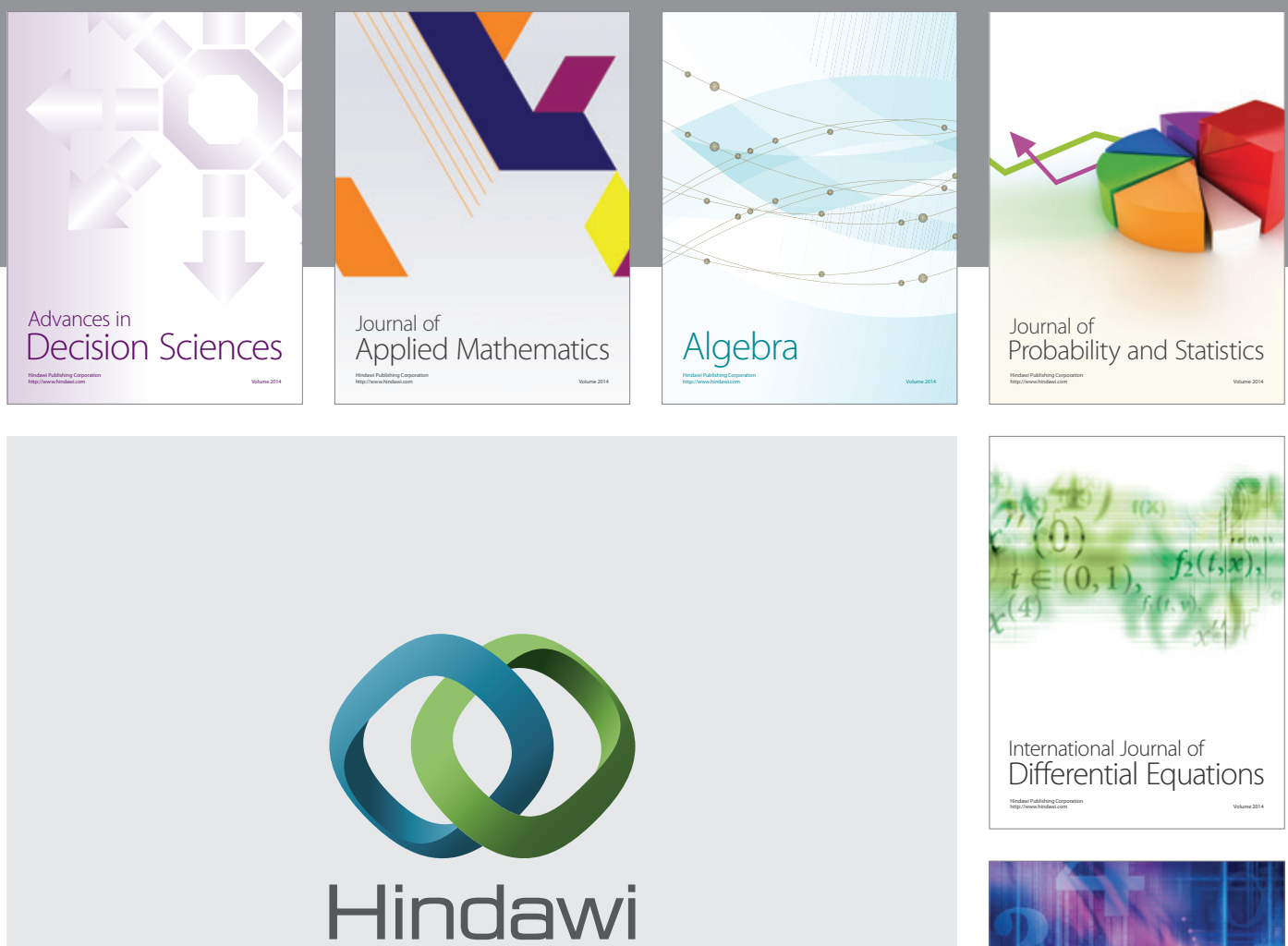

Submit your manuscripts at http://www.hindawi.com
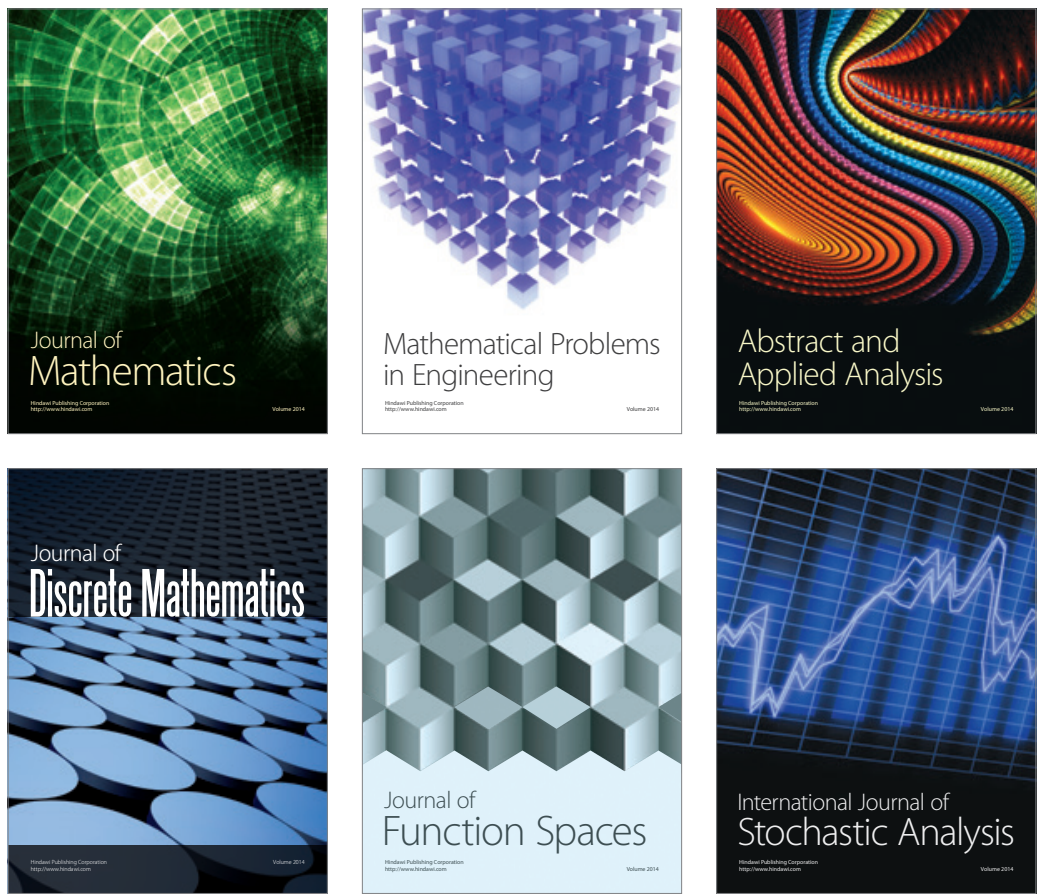

Journal of

Function Spaces

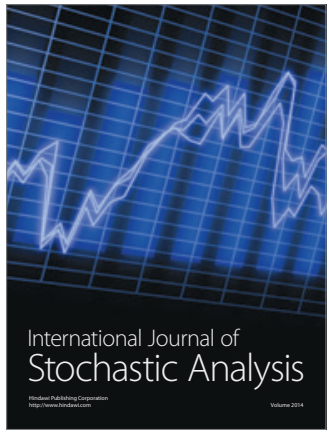

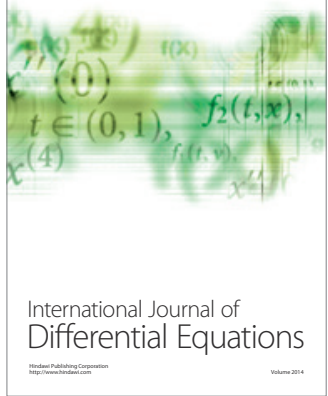
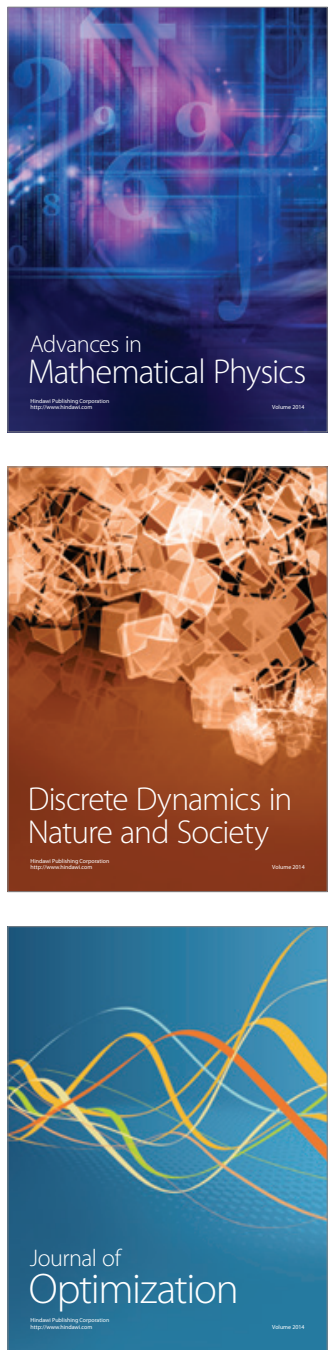